\title{
q-Analogs of Classical 6-Periodicity: From Euler to Chebyshev
}

\author{
Boris A KUPERSHMIDT \\ The University of Tennessee Space Institute, Tullahoma, TN 37388, USA \\ E-mail: bkupersh@utsi.edu
}

Received November 19, 2002; Accepted January 03, 2003

\begin{abstract}
The sequence of period 6 starting with $1,1,0,-1,-1,0$ appears in many different disguises in mathematics. Various $q$-versions of this sequence are found, and their relations with Euler's pentagonal numbers theorem and Chebyshev polynomials are discussed.
\end{abstract}

The motto on Cardinal Newman's tomb ought to be the funeral motto of every Catholic, Ex umbris et imaginibus in veritatem, Out of shadows and appearances into the truth.

Ronald Knox, The Pastoral Sermons

\section{Introduction}

The sequence of period 6 , starting with

$$
1,1,0,-1,-1,0
$$

appears as the coefficients in the expansion

$$
\frac{1}{1-x+x^{2}}=1+x-x^{3}-x^{4}+x^{6}+x^{7}-x^{9}-x^{10}+x^{12}+x^{13}-\cdots .
$$

This is easy to see $([15, \mathrm{Ch} . \mathrm{V}])$ :

$$
\begin{aligned}
\sum_{s=0}^{\infty} a_{s} x^{s} & =\frac{1}{1-x+x^{2}}=\frac{1+x}{1+x^{3}}=\frac{1}{1+x^{3}}+x \frac{1}{1+x^{3}} \\
& =\sum_{\ell=0}^{\infty}\left(-x^{3}\right)^{\ell}+x \sum_{\ell=0}^{\infty}\left(-x^{3}\right)^{\ell},
\end{aligned}
$$

so that

$$
\begin{aligned}
& a_{s+3}=-a_{s}, \quad s \in \mathbb{Z}_{+}, \\
& a_{0}=1, \quad a_{1}=1, \quad a_{2}=0:
\end{aligned}
$$

the 6-periodicity results from 3-antiperiodicity. 
This is so far unremarkable. However,

$$
\begin{aligned}
\frac{1}{1-x+x^{2}} & =\frac{1}{1-x(1-x)}=\sum_{m=0}^{\infty} x^{m}(1-x)^{m} \\
& =\sum_{m \geq 0} x^{m} \sum_{k=0}^{m}\left(\begin{array}{c}
m \\
k
\end{array}\right)(-1)^{k} x^{k}=\sum_{n=0}^{\infty} x^{n} \sum_{k=0}^{\lfloor n / 2\rfloor}(-1)^{k}\left(\begin{array}{c}
n-k \\
k
\end{array}\right) .
\end{aligned}
$$

Thus, the binomial sums

$$
s_{n}=\sum_{k=0}^{\lfloor n / 2\rfloor}(-1)^{k}\left(\begin{array}{c}
n-k \\
k
\end{array}\right)
$$

are equal to

$$
1,1,0,-1,-1,0 \quad \text { for } \quad n \equiv 0,1,2,3,4,5 \quad(\bmod 6) .
$$

Such periodicity is very rare for binomial sums; see some examples and references in $[12$, 13]. The problem is: can this periodicity be quantized, in the sense of $q$-mathematics?

\section{Guessing an answer}

We need to find a suitable $q$-analog of the series $\sum_{m=0}^{\infty} x^{m}(1-x)^{m}$ (1.5a). After some experimenting and rescaling, the following sum suggests itself for consideration:

$$
\begin{aligned}
S(x)=S(x ; q) & =\sum_{m=0}^{\infty} x^{m}(1 \dot{-} x)^{m} \\
& =\sum_{m=0}^{\infty} x^{m}(x ; q)_{m},
\end{aligned}
$$

where

$$
(a \dot{+} b)^{m}=\left[\prod_{i=0}^{m}\left(a+q^{i} b\right)\right] /\left(a+q^{m} b\right)= \begin{cases}(a+b) \cdots\left(a+q^{m-1} b\right), & m \in \mathbb{N} \\ 1, & m=0\end{cases}
$$

and (see [2, p. 487])

$$
(x ; q)_{m}=\left[\prod_{i \geq 0}^{m}\left(1-q^{i} x\right)\right] /\left(1-q^{m} x\right)= \begin{cases}(1-x) \cdots\left(1-q^{m-1} x\right), & m \in \mathbb{N}, \\ 1, & m=0 .\end{cases}
$$

By Euler's formula [2, p. 492],

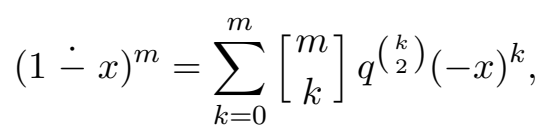


where

$$
\left[\begin{array}{c}
m \\
k
\end{array}\right]=\left[\begin{array}{c}
m \\
k
\end{array}\right]_{q}=\frac{[m] \cdots[m-k+1]}{[1] \cdots[k]}, \quad k \in \mathbb{N} ; \quad\left[\begin{array}{c}
m \\
0
\end{array}\right]=1,
$$

are the $q$-binomial coefficients, and

$$
[m]=[m]_{q}=\left(1-q^{m}\right) /(1-q)
$$

is a $q$-analog of the classical number (or object) $m$.

Substituting (2.4) into (2.1), we get

$$
S(x)=\sum_{m=0}^{\infty} x^{m}(1-x)^{m}=\sum_{n=0}^{\infty} x^{n} \sum_{k=0}^{\lfloor n / 2\rfloor}(-1)^{k}\left[\begin{array}{c}
n-k \\
k
\end{array}\right]_{q} q^{\left(\begin{array}{c}
k \\
2
\end{array}\right)} .
$$

Thus, as a $q$-analog of the classical binomial sum (1.6a) we obtain

$$
S_{n}=\sum_{k=0}^{\lfloor n / 2\rfloor}(-1)^{k}\left[\begin{array}{c}
n-k \\
k
\end{array}\right] q^{\left(\begin{array}{c}
k \\
2
\end{array}\right)}
$$

Is it any good? Calculating a few terms, we find:

$$
S(x)=1+x+0 \cdot x^{2}-q x^{3}-q^{2} x^{4}+0 \cdot x^{5}+q^{5} x^{6}+q^{7} x^{7}+0 \cdot x^{8}+O\left(x^{9}\right) .
$$

This looks promissing, as the 3 -antiperiodicity is preserved here, in $q$-clothes, but there are not enough terms to guess the rule for the $q$-exponents. We need some sort of a functional equation to determine that rule, assuming it exists.

We proceed as follows. Set

$$
S(x, a)=S(x, a ; q)=\sum_{m=0}^{\infty} a^{m} x^{m}(1 \dot{-} x)^{m} .
$$

Since

$$
(1 \dot{-} x)^{m+1}=(1-x)(1 \dot{-} q x)^{m},
$$

we find that

$$
S(x, a)=1+a x(1-x) \sum_{m=0}^{\infty}(a x)^{m}(1 \dot{-} q x)^{m}=1+a x(1-x) S(q x, a / q) .
$$

Therefore, if we write

$$
S(x, a)=\sum_{n=0}^{\infty} S_{n}(a) x^{n},
$$

the functional equation (2.12a) converts itself into

$$
\begin{aligned}
& S_{n+2}(a)=a S_{n+1}(a / q) q^{n+1}-a S_{n}(a / q) q^{n}, \quad n \in \mathbb{Z}_{+}, \\
& S_{1}(a)=a, \quad S_{0}(a)=1 .
\end{aligned}
$$


In particular, for $a=q$, we find:

$$
\begin{aligned}
& S_{0}(q)=1, \quad S_{1}(q)=q, \\
& S_{n+2}(q)=q^{n+1}\left(q S_{n+1}-S_{n}\right), \quad S_{n}=S_{n}(1), \quad n \in \mathbb{Z}_{+} .
\end{aligned}
$$

Using the expansion (2.9), we obtain then

$$
\begin{aligned}
S(x, q)= & \sum_{m=0}^{\infty}(q x)^{m}(1 \dot{-} x)^{m}=1+q x+\left(q^{2}-q\right) x^{2}-q^{2} x^{3}-q^{5} x^{4} \\
& -\left(q^{2}-q^{7}\right) x^{5}+q^{7} x^{6}+q^{12} x^{7}+\left(q^{15}-q^{12}\right) x^{8}+O\left(x^{9}\right) .
\end{aligned}
$$

This is a bit less simple than (2.9) but still looks enticing. Moreover, comparing both expansions we can't fail to notice some coincidences and regularities; the following general ansatz suggests itself:

$$
\begin{array}{ll}
S_{6 n}(q)=q^{x(n)}, & S_{6 n}=q^{x(n)-2 n}, \\
S_{6 n+1}(q)=q^{y(n)}, & S_{6 n+1}=q^{x(n)}, \\
S_{6 n+2}(q)=q^{y(n)}\left(q^{2 n+1}-1\right), & S_{6 n+2}=0, \\
S_{6 n+3}(q)=-q^{u(n)}, & S_{6 n+3}=-q^{u(n)-2 n-1}, \\
S_{6 n+4}(q)=-q^{v(n)}, & S_{6 n+4}=-q^{u(n)}, \\
S_{6 n+5}(q)=-q^{v(n)}\left(q^{2 n+2}-1\right), & S_{6 n+5}=0 .
\end{array}
$$

Making this ansatz compatible with the relations (2.14) leads to the determination of all the unknown exponents $x(n), y(n), u(n), v(n)$ in formulae $(2.16)$ :

$$
\begin{aligned}
& y(n)=x(n)+4 n+1, \\
& u(n)=x(n)+6 n+2, \\
& v(n)=u(n)+4 n+3, \\
& x(n+1)=6 n+5+u(n)
\end{aligned}
$$

$\Rightarrow$

$$
\begin{aligned}
& x(n)=6 n^{2}+n, \\
& y(n)=6 n^{2}+5 n+1, \\
& u(n)=6 n^{2}+7 n+2 \\
& v(n)=6 n^{2}+11 n+5 .
\end{aligned}
$$

These are our two - conjectured so far - candidates for a quantum versions of the 6periodic classical binomial sum (1.6). Are there any other candidates? It seems unlikely, at least if we insist on having relatively compact answers. For example,

$$
\begin{aligned}
& S_{4}(a) / a^{2}=q-a[3]+a^{2}, \\
& S_{5}(a) / a^{3}=q[3]-a[4]+a^{2},
\end{aligned}
$$

and for $a=q^{L}$ we don't get anything attractive unless $L=0$ or 1 . 
Let us now collect our conjectured formulae into a series form. For $a=1$, we get

$$
\begin{aligned}
& S(x)=S(x, 1)=\sum_{n=0}^{\infty} S_{n} x^{n} \\
& =\sum_{n=0}^{\infty}\left(x^{6 n} q^{6 n^{2}-n}+x^{6 n+1} q^{6 n^{2}+n}-x^{6 n+3} q^{6 n^{2}+5 n+1}-x^{6 n+4} q^{6 n^{2}+7 n+2}\right) \\
& =\sum_{n=0}^{\infty}\left\{\left(x^{6 n} q^{6 n^{2}-n}-x^{6 n+3} q^{6 n^{2}+5 n+1}\right)+\left(x^{6 n+1} q^{6 n^{2}+n}-x^{6 n+4} q^{6 n^{2}+7 n+2}\right)\right\} \\
& =\sum_{n=0}^{\infty}(-1)^{n}\left\{x^{3 n} q^{n(3 n-1) / 2}+x^{3 n+1} q^{n(3 n+1) / 2}\right\}: \\
& \sum_{m=0}^{\infty} x^{m}(1 \dot{-} x)^{m}=\sum_{n=0}^{\infty}(-x)^{3 n} q^{n(3 n-1) / 2}\left(1+x q^{n}\right) .
\end{aligned}
$$

Similarly, for $a=q$ we obtain

$$
\begin{aligned}
& S(x, q)=\sum_{n=0}^{\infty} S_{n}(q) x^{n} \\
& =\sum_{n=0}^{\infty} x^{6 n}\left\{q^{6 n^{2}+n}+x q^{6 n^{2}+5 n+1}+x^{2} q^{6 n^{2}+5 n+1}\left(q^{2 n+1}-1\right)\right. \\
& \left.-x^{3} q^{6 n^{2}+7 n+2}-x^{4} q^{6 n^{2}+11 n+5}-x^{5} q^{6 n^{2}+11+5}\left(q^{2 n+2}-1\right)\right\} \\
& =\sum_{n=0}^{\infty}(-1)^{n}\left\{x^{3 n} q^{n(3 n+1) / 2}+x^{3 n+1} q^{n(3 n+5) 2}+x^{3 n+2} q^{n(3 n+5) / 2}\left(q^{n+1}-1\right)\right\}: \\
& \sum_{m=0}^{\infty} q^{m} x^{m}(1-x)^{m}=\sum_{n=0}^{\infty}(-x)^{3 n} q^{n(3 n+1) / 2}\left\{1+x q^{2 n}+x^{2} q^{2 n}\left(q^{n+1}-1\right)\right\} .
\end{aligned}
$$

In view of the functional equation (2.12), formula (2.21) implies formula (2.23). In the next Section we shall prove the former formula.

Remark 2.24. Formula (2.12a) shows that the parameter

$$
y=a x
$$

remains invariant under iteration. Therefore, let us set

$$
R(x, y)=R(x, y ; q)=\sum_{m=0}^{\infty} y^{m}(1 \dot{-} x)^{m} .
$$

Then

$$
R(x, y)=1+y(1-x) R(q x, y) .
$$

Setting

$$
R(x, y)=\sum_{\ell=0}^{\infty} c_{\ell}(y) x^{\ell},
$$


we convert the functional equation (2.27) into

$$
\begin{aligned}
& c_{0}=1+y c_{0}, \\
& c_{\ell+1}=y q^{\ell+1} c_{\ell+1}-y q^{\ell} c_{\ell} \quad \Rightarrow \\
& c_{\ell}=\frac{\left.(-y)^{\ell} q^{(} \begin{array}{l}
\ell \\
2
\end{array}\right)}{(1 \dot{-} y)^{\ell+1}}, \quad \ell \in \mathbb{Z}_{+} \quad \Rightarrow \\
& R(x, y)=\sum_{m=0}^{\infty} y^{m}(1 \dot{-} x)^{m}=\sum_{\ell=0}^{\infty} \frac{(-x y)^{\ell}}{(1 \dot{-} y)^{\ell+1}} q^{\left(\begin{array}{l}
\ell \\
2
\end{array}\right)} \quad \Rightarrow \\
& S(x, a)=\sum_{m=0}^{\infty}(a x)^{m}(1 \dot{-} x)^{m}=\sum_{\ell=0}^{\infty} \frac{\left(-a x^{2}\right)^{\ell}}{(1 \dot{-a x})^{\ell+1}} q^{\left(\begin{array}{l}
\ell \\
2
\end{array}\right) .}
\end{aligned}
$$

Neither of these expansions, however, is helpful for out task of proving formula (2.21). We need something completely different. Notice that for $q=1$, formula (2.31) yields:

$$
\frac{1}{1-y(1-x)}=\sum_{m=0}^{\infty} y^{m}(1-x)^{m}=\sum_{\ell=0}^{\infty} \frac{(-x y)^{\ell}}{(1-y)^{\ell+1}}=\frac{1}{1-y} \frac{1}{1+\frac{x y}{1-y}}
$$

\section{Euler's tower}

We have to prove formula (2.21). In the form (2.20b), it is:

$$
\sum_{m=0}^{\infty} x^{m}(1 \dot{-} x)^{m}=\sum_{n=0}^{\infty}(-1)^{n}\left\{x^{3 n} q^{n(3 n-1) / 2}+x^{3 n+1} q^{n(3 n+1) / 2}\right\} .
$$

This is an identity between formal power series in $x$, with coefficients that are polynomials in $q$. We can not therefore let $x$ to be 1 (unlike $q$ ). But let's get wild for a moment. The RHS of formula (3.1) for $x=1$ is:

$$
\sum_{n=0}^{\infty}(-1)^{n}\left\{q^{n(3 n-1) / 2}+q^{n(3 n+1) / 2}\right\}=1+\sum_{n=-\infty}^{\infty}(-1)^{n} q^{n(3 n+1) / 2} .
$$

This is essentially the RHS of Euler's famous identity, conjectured by him in 1741:

$$
\prod_{j=1}^{\infty}\left(1-q^{j}\right)=\sum_{n=-\infty}^{\infty}(-1)^{n} q^{n(3 n+1) / 2} .
$$

(See [15, Ch. 6] for an English translation of Euler's fascinating memoir.) Nowadays, Euler's formula (3.3) is subsumed by the more general Jacobi triple product identity

$$
\prod_{n=1}^{\infty}\left(1+Q^{2 n-1} z\right)\left(1+Q^{2 n-1} z^{-1}\right)\left(1-Q^{2 n}\right)=\sum_{n=-\infty}^{\infty} Q^{n^{2}} z^{n}
$$

(see [16, p. 10 and p. 186]). Euler's formula (3.3) results from (3.4) when one makes the substitution

$$
Q=q^{3 / 2}, \quad z=-q^{1 / 2} .
$$


Neither Euler's formula (3.3) nor Jacobi's formula (3.4) seem of any help to our identity (3.1). However, Euler's ingenious Proof of his identity (see [24, p. 281]) has enough ingredients in it to establish (3.1). Weil describes Euler's Proof as "another dazzling display of algebraic virtuosity but quite elementary ...".

Euler sets

$$
P_{0}=\prod_{j=1}^{\infty}\left(1-q^{j}\right)
$$

and notices that

$$
P_{0}=1-q-q^{2} P_{1}
$$

where

$$
P_{1}=\sum_{\nu=0}^{\infty} q^{\nu}(1-q) \cdots\left(1-q^{\nu+1}\right)
$$

This follows from the easily proven by induction identity

$$
\prod_{j=1}^{n}\left(1-\alpha_{j}\right)=1-\alpha_{1}-\sum_{k=2}^{n} \alpha_{k}\left(1-\alpha_{1}\right) \cdots\left(1-\alpha_{k-1}\right), \quad n \geq 2
$$

by setting $\alpha_{j}=q^{j}$, replacing $k$ by $\nu+2$, and then passing to the limit $n \rightarrow \infty$. Euler then introduces the infinite tower of series $P_{n}$ :

$$
P_{n}=\sum_{\nu=0}^{\infty} q^{\nu n}\left(1-q^{n}\right) \cdots\left(1-q^{n+\nu}\right), \quad n \in \mathbb{N},
$$

and shows by a different argument that

$$
P_{n}=1-q^{2 n+1}-q^{3 n+2} P_{n+1}, \quad n \in \mathbb{N} .
$$

Euler's argument for $n>0$ is ingenious:

$$
\begin{aligned}
& P_{n}=\sum_{\nu=0}^{\infty} q^{\nu n}\left(1-q^{n}\right) \cdots\left(1-q^{n+\nu}\right) \\
& =\left(1-q^{n}\right)+q^{n}\left(1-q^{n}\right)\left(1-q^{n+1}\right)+\sum_{\mu=2}^{\infty} q^{\mu n}\left(1-q^{n}\right) \cdots\left(1-q^{n+\mu}\right) \\
& =1-q^{n}+q^{n}\left(1-q^{n+1}\right)-q^{2 n}\left(1-q^{n+1}\right) \\
& +\sum_{\mu=2}^{\infty} q^{\mu n}\left(1-q^{n+1}\right) \cdots\left(1-q^{n+\mu}\right)-q^{n} \sum_{\mu=2}^{\infty} q^{\mu n}\left(1-q^{n+1}\right) \cdots\left(1-q^{n+\mu}\right)
\end{aligned}
$$




$$
\begin{aligned}
= & 1-q^{2 n+1}-q^{2 n}\left(1-q^{n+1}\right)+\sum_{\nu=0}^{\infty} q^{\nu n+2 n}\left(1-q^{n+1}\right) \cdots\left(1-q^{n+2+\nu}\right) \\
& -\sum_{\mu=1}^{\infty} q^{(\mu+1) n}\left(1-q^{n+1}\right) \cdots\left(1-q^{n+\mu}\right)+q^{2 n}\left(1-q^{n+1}\right) \\
= & 1-q^{2 n+1}+\sum_{\nu=0}^{\infty} q^{\nu(n+1)+2 n-\nu}\left(1-q^{n+1}\right) \cdots\left(1-q^{n+1+\nu}\right)\left(1-q^{n+2+\nu}\right) \\
& -\sum_{\nu=0}^{\infty} q^{(\nu+2) n}\left(1-q^{n+1}\right) \cdots\left(1-q^{n+1+\nu}\right) \\
= & 1-q^{2 n+1}+\sum_{\nu=0}^{\infty} q^{\nu(n+1)}\left(1-q^{n+1}\right) \cdots\left(1-q^{n+1+\nu}\right)\left\{q^{2 n-\nu}\left(1-q^{n+2+\nu}\right)-q^{2 n-\nu}\right\} \\
= & 1-q^{2 n+1}-q^{3 n+2} \sum_{\nu=0}^{\infty} q^{\nu(n+1)}\left(1-q^{n+1}\right) \cdots\left(1-q^{n+1+\nu}\right)=1-q^{2 n+1}-q^{3 n+2} P_{n+1} .
\end{aligned}
$$

Collecting all the relations (3.11) together, we find

$$
\begin{aligned}
& \prod_{j=1}^{\infty}\left(1-q^{j}\right)=P_{0}=1-q-q^{2} P_{1}=1-q-q^{2}\left(1-q^{3}-q^{5} P_{2}\right) \\
& =1-q-q^{2}\left(1-q^{3}\right)+q^{2+5}\left(1-q^{5}-q^{8} P_{3}\right)=\cdots \\
& =1-q+\sum_{n=1}^{\infty}(-1)^{n} q^{2+5+\cdots+(3 n-1)}\left(1-q^{2 n+1}\right) \\
& =1-q+\sum_{n=1}^{\infty}(-1)^{n} q^{n(3 n+1) / 2}\left(1-q^{2 n+1}\right)=\sum_{n=0}^{\infty}(-1)^{n} q^{n(3 n+1) / 2}\left(1-q^{2 n+1}\right) \\
& =\sum_{n=0}^{\infty}(-1)^{n} q^{n(3 n+1) / 2}+\sum_{n=0}^{\infty}(-1)^{n+1} q^{\left(3 n^{2}+n+4 n+2\right) / 2}=\sum_{n=-\infty}^{\infty}(-1)^{n} q^{n(3 n+1) / 2} .
\end{aligned}
$$

While we are at it, let's notice that a similar iteration yields

$$
P_{\ell}=\sum_{n=0}^{\infty}(-1)^{n} q^{3 \ell n+n(3 n+1) / 2}\left(1-q^{2 \ell+2 n+1}\right), \quad \ell \in \mathbb{Z}_{+} .
$$

From this, we can readily see that

$$
\bar{P}_{\ell}:=1+q^{\ell} P_{\ell}=\sum_{n=0}^{\infty}(-1)^{n}\left\{q^{3 \ell n} q^{n(3 n-1) / 2}+q^{(3 n+1) \ell} q^{n(3 n+1) / 2}\right\} .
$$

It now only remains to compare $\bar{P}_{\ell}$ with

$$
\begin{aligned}
& \bar{S}_{\ell}=S\left(q^{\ell} ; q\right)=\left.S(x ; q)\right|_{x=q^{\ell}}, \quad \forall \ell \in \mathbb{N}: \\
& \bar{S}_{\ell}=S\left(q^{\ell} ; q\right)=\sum_{m=0}^{\infty} q^{\ell m}\left(1 \dot{-} q^{\ell}\right)^{m}=1+\sum_{m=1}^{\infty} q^{\ell m}\left(1-q^{\ell}\right) \cdots\left(1-q^{\ell+m-1}\right), \\
& \bar{P}_{\ell}=1+q^{\ell} \sum_{\nu=0}^{\infty} q^{\nu \ell}\left(1-q^{\ell}\right) \cdots\left(1-q^{\ell+\nu}\right),
\end{aligned}
$$


and these are identical. Thus,

$$
\bar{S}_{\ell}=\bar{P}_{\ell}, \quad \ell \in \mathbb{N} .
$$

Comparing formulae (3.1) and (3.16), we get

$$
\begin{aligned}
& \left.\left\{\sum_{m=0}^{\infty} x^{m}(1 \dot{-} x)^{m}\right\}\right|_{x=q^{\ell}} \\
& \quad=\left.\left(\sum_{n=0}^{\infty}(-1)^{n}\left\{x^{3 n} q^{n(3 n-1) / 2}+x^{3 n+1} q^{n(3 n+1) / 2}\right\}\right)\right|_{x=q^{\ell}}, \quad \forall \ell \in \mathbb{N} .
\end{aligned}
$$

This proves formula (3.1), because:

Lemma 3.21. Suppose $f \in \mathbb{C}[[x, y]]$ is formal power series in $x, y$, and let $r(1), r(2), \ldots$ be an increasing sequence of positive intergers. If

$$
f\left(y^{r(i)}, y\right)=\left.f(x, y)\right|_{x=y^{r(i)}}=0, \quad \forall i \in \mathbb{N},
$$

then $f$ is identically zero.

Proof. If $f\left(y^{r(1)}, y\right)=0$ then $f$ is divisible by $x-y^{r(1)}$ :

$$
f(x, y)=\left(x-y^{r(1)}\right) f_{1}(x, y) .
$$

Continuing on, we find that

$$
f(x, y)=\left\{\prod_{i=1}^{k}\left(x-y^{r(i)}\right)\right\} f_{k}(x, y)
$$

Thus, all homogeneous components of $f$ of degrees $<k$ vanish, and $k$ is arbitrary.

\section{Euler's tower revisited}

Woman: Your're a pugilist, arent't you?

World champ boxer Rocky Graziano: Nah, I'm just a prizefighter.

The Euler relation (3.11)

$$
P_{n}=1-q^{2 n+1}-q^{3 n+2} P_{n+1}, \quad n \in \mathbb{N},
$$

for the sequence of series

$$
P_{n}=\sum_{\nu=0}^{\infty} q^{\nu n}\left(1-q^{n}\right) \cdots\left(1-q^{n+\nu}\right), \quad n \in \mathbb{N},
$$

has an implicit gem hidden inside. To make it explicit, notice that in the definition of $P_{n}$ (4.2) the index $n$ could be treated as a formal parameter and not necessarily as a positive integer. So, set

$$
q^{n}=x
$$


and define

$$
\mathcal{P}(x)=\mathcal{P}(x ; q)=\sum_{\nu=0}^{\infty} x^{\nu}(1-x) \cdots\left(1-x q^{\nu}\right) .
$$

Applying the Euler argument to $\mathcal{P}(x)$, we get:

$$
\begin{aligned}
& \mathcal{P}(x)=\sum_{\nu=0}^{\infty} x^{\nu}(1-x) \cdots\left(1-x q^{\nu}\right)=(1-x)+x(1-x)(1-x q) \\
& +\sum_{\mu=2}^{\infty} x^{\mu}(1-x) \cdots\left(1-x q^{\mu}\right)=(1-x)+x(1-x q)-x^{2}(1-x q) \\
& +\sum_{\mu=2}^{\infty} x^{\mu}(1-x q) \cdots\left(1-x q^{\mu}\right)-x \sum_{\mu=2}^{\infty} x^{\mu}(1-x q) \cdots\left(1-x q^{\mu}\right) \\
& =1-q x^{2}-x^{2}(1-x q)+\sum_{\nu=0}^{\infty} x^{\nu+2}(1-x q) \cdots\left(1-x q^{\nu+1}\right)\left(1-x q^{\nu+2}\right) \\
& -\sum_{\mu=1}^{\infty} x^{\mu+1}(1-x q) \cdots\left(1-x q^{\mu}\right)+x^{2}(1-x q) \\
& =1-q x^{2}+x^{2} \sum_{\nu=0}^{\infty} x^{\nu}(1-x q) \cdots\left(1-x q^{\nu+1}\right)\left\{\left(1-x q^{\nu+2}\right)-1\right\} \\
& =1-q x^{2}-x^{3} q^{2} \sum_{\nu=0}^{\infty}(x q)^{\nu}(1-x q) \cdots(1-x q \cdot q)^{\nu}=1-q x^{2}-q^{2} x^{3} \mathcal{P}(x q): \\
& \mathcal{P}(x ; q)=1-q x^{2}-q^{2} x^{3} \mathcal{P}(x q ; q) .
\end{aligned}
$$

Writing

$$
\mathcal{P}(x ; q)=\sum_{\ell=0}^{\infty} c_{\ell}(q) x^{\ell},
$$

we can transform the relation (4.6) into

$$
\begin{aligned}
& c_{\ell+3}=-q^{\ell+2} c_{\ell}, \\
& c_{0}=1, \quad c_{1}=0, \quad c_{2}=-q \quad \Rightarrow \\
& c_{3 \ell}=(-1)^{\ell} q^{\ell(3 \ell+1) / 2}, \\
& c_{3 \ell+1}=0, \\
& c_{3 \ell-1}=(-1)^{\ell} q^{\ell(3 \ell-1) / 2} \quad \Rightarrow \\
& \mathcal{P}(x)=\sum_{\nu=0}^{\infty} x^{\nu}(1-x) \cdots\left(1-x q^{\nu}\right) \\
& \quad=1+\sum_{\ell=1}^{\infty}(-1)^{\ell}\left\{q^{\ell(3 \ell-1) / 2} x^{3 \ell-1}+q^{\ell(3 \ell+1) / 2} x^{3 \ell}\right\} .
\end{aligned}
$$


If we notice that

$$
S(x)=1+x \mathcal{P}(x),
$$

then from formula $(4.10 \mathrm{~b})$ we obtain at once that

$$
\begin{aligned}
S(x) & =\sum_{m=0}^{\infty} x^{m}(1 \dot{-} x)^{m} \\
& =\sum_{\ell=0}^{\infty}(-1)^{\ell}\left\{x^{3 \ell} q^{\ell(3 \ell-1) / 2}+x^{3 \ell+1} q^{\ell(3 \ell+1) / 2}\right\},
\end{aligned}
$$

which is our conjectured formula (2.20b). The functional equation (4.6) in the $S$-language becomes:

$$
S(x)=1+x-q x^{3} S(q x) ;
$$

this equation by itself implies formula (4.12b) at once.

Remark 4.14. The identity (4.10) is essentially due to Euler, but it was repeatedly rediscovered many times since. See $[1$, p. 282] for more on that.

Remark 4.15. What is the orgin of number 6 , and are there interesting periodic sequences of period other than 6? I don't yet have a definite answer to these questions but suspect that our 6-periodic sequence is a camouflaged group of units of the field $\mathbb{Q}[\sqrt{-3}]$; this suggests that there exists a 4 -periodic sequence attached to the units $\pm 1, \pm i$ of $\mathbb{Q}[\sqrt{-1}]$, and if a number field $K \supset \mathbb{Q}$ has a finite group of units then these can be organized into a periodic (vector) sequence.

\section{Recurrence relations of second order}

The space of 3-antiperiodic sequences is 3-dimensional. It contains a special 2-dimensional subspace consisting of $2^{n d}$-order recurrent sequences $\left\{u_{n}\right\}$ satisfying the relation

$$
u_{n+2}=u_{n+1}-u_{n} .
$$

Indeed, for $u_{0}=a, u_{1}=b$, the sequence $u_{n}$ starts as

$$
a, b, b-a,-a,-b,-b+a \mid a, b, \ldots
$$

(In the language of characteristic polynomials: $\lambda^{3}+1$ is divisible by $\lambda^{2}-\lambda+1$ ).

Our sequence of binomial sums $s_{n}$ (1.6a)

$$
s_{n}=\sum_{k=0}^{\lfloor n / 2\rfloor}(-1)^{k}\left(\begin{array}{c}
n-k \\
k
\end{array}\right)
$$

is of this special form, with $a=b=1$. This is true a posteriori, from comparing formulae (5.2) and (1.6b), but can be easily seen directly from the definition of $s_{n}$ (5.3):

$$
\begin{gathered}
s_{n+2}-s_{n+1}=\sum_{k \geq 0}(-1)^{k}\left\{\left(\begin{array}{c}
n+2-k \\
k
\end{array}\right)-\left(\begin{array}{c}
n+1-k \\
k
\end{array}\right)\right\}=\sum(-1)^{k}\left(\begin{array}{c}
n+1-k \\
k-1
\end{array}\right) \\
=\sum(-1)^{k+1}\left(\begin{array}{c}
n-k \\
k
\end{array}\right)=-s_{n} .
\end{gathered}
$$


In quantizing $s_{n}$ in $\S 2$, we went along the route of generating functions, quantizing the latter for the sequence $s_{n}$ :

$$
\sum_{n=0}^{\infty} s_{n} x^{n}=\sum_{m=0}^{\infty} x^{m}(1-x)^{m} .
$$

Generating functions make a very useful and powerful device (see [25].) However, when one attempts to quantize a classical object, property, relation, etc., the generating function methods may unnecessarily restrict one's choice. A cautionary tale follows.

Let's try to quantize the calculation (5.4). Set

$$
f_{n}=f_{n \mid L}=f_{n \mid L}(q)=\sum_{k=0}^{\lfloor n / 2\rfloor}\left(-q^{L}\right)^{k} q^{\theta(n, k)}\left[\begin{array}{c}
n-k \\
k
\end{array}\right]_{q}
$$

with some unknown function $\theta(n, k)$ to be specified later on. We shall use the formulae

$$
\begin{aligned}
{\left[\begin{array}{c}
\alpha+1 \\
r
\end{array}\right] } & =q^{r}\left[\begin{array}{l}
\alpha \\
r
\end{array}\right]+\left[\begin{array}{c}
\alpha \\
r-1
\end{array}\right] \\
& =\left[\begin{array}{l}
\alpha \\
r
\end{array}\right]+q^{\alpha+1-r}\left[\begin{array}{c}
\alpha \\
r-1
\end{array}\right] .
\end{aligned}
$$

First, by formula (5.7a)

$$
\begin{aligned}
& f_{n+2 \mid L}=\sum_{k}\left(-q^{L}\right)^{k} q^{\theta(n+2, k)}\left[\begin{array}{c}
n+2-k \\
k
\end{array}\right] \\
& =\sum_{k}\left(-q^{L}\right)^{k} q^{\theta(n+2, k)}\left\{q^{k}\left[\begin{array}{c}
n+1-k \\
k
\end{array}\right]+\left[\begin{array}{c}
n+1-k \\
k-1
\end{array}\right]\right\} \\
& =\sum\left(-q^{L+1}\right)^{k} q^{\theta(n+2, k)}\left[\begin{array}{c}
n+1-k \\
k
\end{array}\right]-q^{L} \sum\left(-q^{L}\right)^{k} q^{\theta(n+2, k+1)}\left[\begin{array}{c}
n-k \\
k
\end{array}\right] .
\end{aligned}
$$

This is an opaque expression. If we simplify it by having $L=0$, demanding that

$$
\theta(n+2, k)+k=\theta(n+1, k)
$$

modulo rescaling of $f_{n}$ by a function of $n$, and then requiring that

$$
\{\theta(n+2, k+1)-\theta(n, k)\} \quad \text { is } k \text {-independent, }
$$

we find that

$$
\theta(n, k)=k(k-n),
$$

and formula (5.8) yields:

$$
f_{n+2}=f_{n+1}-q^{-(n+1)} f_{n} .
$$

Changing $q$ into $q^{-1}$, we get:

$$
\begin{aligned}
& f_{n+2}=f_{n+1}-q^{n+1} f_{n}, \quad f_{0}=f_{1}=1, \\
& f_{n}=\sum_{k=0}^{\lfloor n / 2\rfloor}(-1)^{k} q^{k^{2}}\left[\begin{array}{c}
n-k \\
k
\end{array}\right]_{q}
\end{aligned}
$$


because

$$
\begin{aligned}
& {[n] !_{q-1}=[n] ! q^{-\left(\begin{array}{c}
n \\
2
\end{array}\right)},} \\
& {\left[\begin{array}{l}
n \\
r
\end{array}\right]_{q^{-1}}=\left[\begin{array}{l}
n \\
r
\end{array}\right]_{q} q^{r(r-n)} .}
\end{aligned}
$$

The sequence $f_{n}$ is:

$$
\begin{aligned}
& 1,1,1-q, 1-q-q^{2}, 1-q-q^{2}-q^{3}+q^{4}, 1-q-q^{2}-q^{3}+q^{5}+q^{6}, \\
& 1-q-q^{2}-q^{3}+2 q^{6}+q^{7}+q^{8}+q^{9}, \ldots
\end{aligned}
$$

and it doesn't appear interesting; however, its limit $f_{\infty}$ certainly is: from formula (5.14) with $n=\infty$, we get

$$
f_{\infty}=\sum_{k=0}^{\infty} \frac{(-1)^{k} q^{k^{2}}}{(1 \dot{-} q)^{k}} .
$$

Secondly, if we use formula (5.7b) instead of (5.7a), we find (with $L=0$ ):

$$
\begin{aligned}
& f_{n+2}=\sum(-1)^{k} q^{\theta(n+2, k)}\left\{\left[\begin{array}{c}
n+1-k \\
k
\end{array}\right]+q^{n+2-2 k}\left[\begin{array}{c}
n+1-k \\
k-1
\end{array}\right]\right\} \\
& =\sum(-1)^{k} q^{\theta(n+2, k)}\left[\begin{array}{c}
n+1-k \\
k
\end{array}\right]-q^{n} \sum(-1)^{k} q^{\theta(n+2, k+1)-2 k}\left[\begin{array}{c}
n-k \\
k
\end{array}\right] .
\end{aligned}
$$

The first summand in (5.17) implies that $\theta(n, k)$ is $n$-independent, and the second summand yields

$$
\theta(n, k)=k^{2}
$$

Thus,

$$
\begin{aligned}
& f_{n+2}=f_{n+1}-q^{n+1} f_{n}, \quad f_{0}=f_{1}=1, \\
& f_{n}=\sum_{k=0}^{\lfloor n / 2\rfloor}(-1)^{k} q^{k^{2}}\left[\begin{array}{c}
n-k \\
k
\end{array}\right]_{q},
\end{aligned}
$$

which is the same as formula (5.14).

If we set

$$
F(x)=\sum_{n=0}^{\infty} f_{n} x^{n},
$$

then formula (5.19) converts itself into

$$
F(x)(1-x)=1-q x^{2} F(q x),
$$

whence, by repeated iteration,

$$
F(x)=\sum_{k=0}^{\infty} \frac{\left(-x^{2}\right)^{k} q^{k^{2}}}{(1 \dot{-} x)^{k+1}} .
$$


Our original sequence $S_{n}(2.8)$,

$$
S_{n}=\sum_{k=0}^{\lfloor n / 2\rfloor}(-1)^{k}\left[\begin{array}{c}
n-k \\
k
\end{array}\right] q^{\left(\begin{array}{c}
k \\
2
\end{array}\right)}
$$

satisfies, as can be directly checked, the following $q$-analog of the relation (5.1):

$$
S_{n}=q^{\lfloor n / 3\rfloor} S_{n-1}-q^{\lfloor 2 n / 3\rfloor-1} S_{n-2} ;
$$

the more general sequence $S_{n}(a)(2.12)$ :

$$
S_{n}(a)=\sum_{k=0}^{\lfloor n / 2\rfloor}(-1)^{k}\left[\begin{array}{c}
n-k \\
k
\end{array}\right] q^{\left(\begin{array}{c}
k \\
2
\end{array}\right) a^{n-k},}
$$

for $a=q$ satisfies the easily verified relation

$$
S_{n}(q)=q^{\lfloor(n+2) / 3\rfloor} S_{n-1}(q)-q^{\lfloor(2 n+1) / 3\rfloor} S_{n-2}(q) .
$$

\section{Chebyshev polynomials and series}

The special 3-antiperiodic relation (5.1)

$$
u_{n+2}=u_{n+1}-u_{n}
$$

is satisfied by the values of the Chebyshev polynomials $T_{n}(x)$ and $U_{n}(x)$ at $x=1 / 2$. Let us recall the salient facts.

Originally, Chebyshev polynomials appeared as the least deviant monic polynomials of a fixed degree, $n$ say, on the interval $-1 \leq x \leq 1$. For the norm

$$
\|f\|=\max _{-1 \leq x \leq 1}|f(x)|
$$

the answer is $T_{n}(x) / 2^{n-1}$,

$$
T_{n}(x)=\cos (n \theta), \quad x=\cos \theta,
$$

being the Chebyshev polynomial of the $1^{\text {st }}$ kind; for the norm

$$
\|f\|=\int_{-1}^{1}|f(x)| d x
$$

the answer is $U_{n}(x) / 2^{n}$,

$$
U_{n}(x)=\frac{\sin ((n+1) \theta))}{\sin \theta}, \quad x=\cos \theta,
$$

being the Chebyshev polynomial of the $2^{\text {nd }}$ kind. 
The following table is extracted from [11, p. 287]:

$$
\begin{aligned}
& T_{0}(x)=1, \\
& T_{1}(x)=x, \\
& T_{2}(x)=2 x^{2}-1, \\
& T_{3}(x)=4 x^{3}-3 x, \\
& T_{4}(x)=8 x^{4}-8 x^{2}+1, \\
& T_{5}(x)=16 x^{5}-20 x^{3}+5 x, \\
& T_{6}(x)=32 x^{6}-48^{4}+18 x^{2}-1, \\
& T_{7}(x)=64 x^{7}-112 x^{5}+56 x^{3}-7 x, \\
& T_{8}(x)=128 x^{8}-256 x^{6}+160 x^{4}-32 x^{2}+1, \\
& T_{9}(x)=256 x^{9}-576 x^{7}+232 x^{5}-120^{3}+9 x ; \\
& U_{0}(x)=1, \\
& U_{1}(x)=2 x \\
& U_{2}(x)=4 x^{2}-1, \\
& U_{3}(x)=8 x^{3}-4 x, \\
& U_{4}(x)=16 x^{4}-12 x^{2}+1, \\
& U_{5}(x)=32 x^{5}-32 x^{3}+6 x, \\
& U_{6}(x)=64 x^{6}-80 x^{4}+24 x^{2}-1, \\
& U_{7}(x)=128 x^{7}-192 x^{5}+80 x^{3}-8 x, \\
& U_{8}(x)=256 x^{8}-448 x^{6}+240 x^{4}-40 x^{2}+1, \\
& U_{9}(x)=512 x^{9}-1024 x^{7}+672 x^{5}-106 x^{3}+10 x .
\end{aligned}
$$

The table (6.4) suggests that

$$
T_{p}(x) \equiv x^{p} \quad(\bmod p), \quad p \text { an odd prime }
$$

which is true because of the easily verifiable formula

$$
T_{n}(x)=\sum_{k=0}^{\lfloor n / 2\rfloor}\left(\begin{array}{c}
n \\
2 k
\end{array}\right) x^{n-2 k}\left(x^{2}-1\right)^{k},
$$

and this interesting congruence is listed as an exercise in [2, p. 117]; there are more exercises on that page, about other interesting arithmetic properties of the Chebyshev polynomials of the $1^{\text {st }}$ and $2^{\text {nd }}$ kind. Some others properties not on that page are:

$$
\begin{array}{ll}
T_{p^{2}}(x) \equiv T_{p}\left(x^{p}\right) \quad\left(\bmod p^{2}\right), & p \text { an odd prime, } \\
T_{2^{n+1}}(x) \equiv 1 \quad\left(\bmod 2^{2 n+1}\right), & n \in \mathbb{Z}_{+},
\end{array}
$$

which follow from formula (6.6) and formula (6.17) below. No doubt there exist many more interesting arithmetic properties of the Chebyshev polynomials. 
Such as. Define the sequence $\left\{\gamma_{n}\right\}$ by the rule:

$$
\gamma_{n+2}=2 \gamma_{n+1}+\gamma_{n}, \quad \gamma_{0}=0, \quad \gamma_{1}=1 .
$$

Then

$$
\begin{aligned}
& T_{2 n}( \pm \sqrt{-1})=1+(-1)^{n} \gamma_{n}^{2}, \quad n \in \mathbb{Z}_{+}, \\
& T_{2 n+1}( \pm \sqrt{-1})= \pm \sqrt{-1}\left\{-1+(-1)^{n}\left(\gamma_{n+1}^{2}-\gamma_{n}^{2}\right) / 2\right\}, \quad n \in \mathbb{Z}_{+} .
\end{aligned}
$$

Most trigonometric formulae translate into formulae for the Chebyshev polynomials in view of the representations (6.2) and (6.3). For example, formula

$$
\cos u+\cos v=2 \cos \frac{u+v}{2} \cos \frac{u-v}{2},
$$

for $u=(n+2) \theta, v=n \theta$; yields

$$
T_{n+2}(x)=2 x T_{n+1}(x)-T_{n}(x) ;
$$

formula

$$
\sin u+\sin v=2 \sin \frac{u+v}{2} \cos \frac{u-v}{2}
$$

yields

$$
U_{n+1}(x)=2 x U_{n}(x)-U_{n-1}(x)
$$

for $u=(n+2) \theta, v=n \theta$; and

$$
U_{n}(x)-U_{n-2}(x)=2 T_{n}(x)
$$

for $u=(n+1) \theta, v=-(n-1) \theta$. (Many more formulae involving Chebyshev polynomials can be found in $[3, \S 10.11],[17,7]$, and $[14, \S 5.7]$.)

Formulae (6.10) for $x=1 / 2$ show that $\left\{T_{n}(1 / 2)\right\}$ and $\left\{U_{n}(1 / 2)\right\}$ are 3 -antiperiodic sequences of the form (6.1), with

$$
\begin{array}{ll}
T_{0}(1 / 2)=1, & T_{1}(1 / 2)=1 / 2, \\
U_{0}(1 / 2)=1, & U_{1}(1 / 2)=1 .
\end{array}
$$

The preceding Sections thus have dealt with quantum aspects of Chebyshev polynomials $U_{n}(x)$ 's at just one point $x=1 / 2$. We won't attempt anything quantum in this Section, as this would be a rather formidable undertaking that is better left to the interested reader. Instead, we shall explore the problem of interpolating the function $u(n)=u_{n}$ from integers to real and complex numbers.

We need a few more standard formulae.

Set temporatily $\tilde{U}_{n}(x)=(n+1)^{-1} d T_{n+1} / d x$. Differentiating formula (6.10a), we get:

$$
(n+1)\left(\tilde{U}_{n+1}-2 x \tilde{U}_{n}+\tilde{U}_{n-1}\right)+\left(\tilde{U}_{n+1}-\tilde{U}_{n-1}-2 T_{n}\right)=0 .
$$


This equation defines $\tilde{U}_{n}$ 's recursively. From the table (6.4),

$$
\begin{aligned}
& \tilde{U}_{0}=T_{1}^{\prime}=1=U_{0}, \\
& \tilde{U}_{1}=T_{2}^{\prime} / 2=2 x=U_{1},
\end{aligned}
$$

and the $U_{n}$ 's also satisfy the equation (6.13) by formulae (6.10b) and (6.11). Therefore, $\tilde{U}_{n}=U_{n}$ :

$$
U_{n}(x)=\frac{1}{n+1} \frac{d T_{n+1}}{d x} .
$$

(Alternatively, one gets formula (6.15) by differentiating formula (6.2) with respect to $x$.) Formulae (6.10) and (6.15) yield:

$$
\begin{aligned}
& T_{n}(1)=1, \\
& U_{n}(1)=n+1, \\
& T_{n}^{\prime}(1)=n^{2} .
\end{aligned}
$$

Formula (6.2) implies that ([19, p. 45], [21, 27])

$$
T_{n}\left(T_{m}(x)\right)=T_{n m}(x), \quad n, m \in \mathbb{N} .
$$

Thus, the polynomials $T_{n}$ 's form a commutative semigroup; below we shall see that this semigroup is in fact a discrete part of a commutative 1-dimensional group considered as either a formal group or as a group of holomorphic automorphisms of a neighborhood of the complex plane around $z=1$. Formula (6.15) implies that $\left\{T_{n}\right.$ 's $\}$ are a more fundamental object than $\left\{U_{n}\right.$ 's $\}$.

Now, from the definition (6.2),

$$
\frac{d^{2} T_{n}}{d \theta^{2}}=-n^{2} T_{n}
$$

Since

$$
\frac{d}{d \theta}=-\sin \theta \frac{d}{d x}, \quad \frac{d^{2}}{d \theta^{2}}=\left(1-x^{2}\right) \frac{d^{2}}{d x^{2}}-x \frac{d}{d x},
$$

we get the classical equation

$$
\left(1-x^{2}\right) T_{n}^{\prime \prime}-x T_{n}^{\prime}+n^{2} T_{n}=0 .
$$

The $T_{n}$ is the unique regular solution of the equation

$$
\left(1-x^{2}\right) y^{\prime \prime}-x y^{\prime}+n^{2} y=0
$$

satisfying the initial condition

$$
y(1)=1 .
$$

In the variable

$$
z=\frac{1-x}{2},
$$


the equation (6.20a) takes the hypergeometric form

$$
z(1-z) \frac{d^{2} y}{d z^{2}}+\left(\frac{1}{2}-z\right) \frac{d y}{d z}+n^{2} y=0,
$$

and we recover another classical result:

$$
T_{n}(x)=F\left(-n, n, \frac{1}{2} ; \frac{1-x}{2}\right) .
$$

(The point $x=\frac{1}{2}$ directs us to the continuous 3 -antiperiodic family $u(\alpha)=F\left(-\alpha, \alpha, \frac{1}{2} ; \frac{1}{4}\right)$.)

Formulae (6.4), (6.5), (6.10) imply that

$$
\begin{aligned}
& T_{n}(0)=U_{n}(0)=0, \quad n \text { odd; } \\
& T_{2 n}(0)=U_{2 n}(0)=(-1)^{n} .
\end{aligned}
$$

The differential equation (6.19) then yields

$$
T_{n}(x)=\frac{n}{2} \sum_{k=0}^{\lfloor n / 2\rfloor}(-1)^{k} \frac{(n-k-1) !}{k !(n-2 k) !}(2 x)^{n-2 k}, \quad n \in \mathbb{N},
$$

and then formula (6.15) yields

$$
U_{n}(x)=\sum_{k=0}^{\lfloor n / 2\rfloor}(-1)^{k}\left(\begin{array}{c}
n-k \\
k
\end{array}\right)(2 x)^{n-2 k}, \quad n \in \mathbb{Z}_{+} ;
$$

another classical pair of very useful formulae.

We now extend the index $n$ in $T_{n}(x)$ by allowing $n$ to be an arbitrary complex number or a formal parameter: we set

$$
T_{\alpha}(x)=\sum_{k=0}^{\infty} c_{\alpha \mid k}(x-1)^{k}, \quad c_{\alpha \mid 0}=1,
$$

and require $T_{\alpha}$ to satisfy the differential equation

$$
\left(1-x^{2}\right) T_{\alpha}^{\prime \prime}-x T_{\alpha}^{\prime}+\alpha^{2} T_{\alpha}=0
$$

together with the boundary condition

$$
T_{\alpha}(1)=1 .
$$

This is equivalent to the relations

$$
\begin{aligned}
& c_{\alpha \mid k+1}=\frac{\alpha^{2}-k^{2}}{(k+1)(2 k+1)} c_{\alpha \mid k}, \quad k \in \mathbb{Z}_{+} \Rightarrow \\
& c_{\alpha \mid k+1}=\frac{1}{(k+1) !(2 k+1) ! !} \prod_{i=0}^{k}\left(\alpha^{2}-i^{2}\right), \quad c_{\alpha \mid 0}=1 .
\end{aligned}
$$


Since

$$
\lim _{k \rightarrow \infty}\left|c_{\alpha \mid k+1} / c_{\alpha \mid k}\right|=1 / 2,
$$

the series $T_{\alpha}(x)(6.27)$ converges for

$$
|x-1|<2 \text {. }
$$

The series terminates only when $\alpha$ is an integer. It is readily verified that

$$
T_{\alpha+2}-2(1+(x-1)) T_{\alpha+1}+T_{\alpha}=0, \quad \forall \alpha,
$$

and that

$$
U_{\alpha}-U_{\alpha-2}=2 T_{\alpha}, \quad \forall \alpha,
$$

where

$$
U_{\alpha}(x)=\frac{1}{\alpha+1} \frac{d T_{\alpha}}{d x}, \quad \forall \alpha .
$$

From this it follows that

$$
U_{\alpha+2}-2\left((1+(x-1)) U_{\alpha+1}+U_{\alpha}=0, \quad \forall \alpha .\right.
$$

Also, formula (6.30) shows that

$$
T_{-\alpha}=T_{\alpha}, \quad \forall \alpha .
$$

Finally,

$$
T_{\alpha}\left(T_{\beta}(x)\right)=T_{\alpha \beta}(x), \quad \forall \alpha, \beta .
$$

Indeed, the difference

$$
T_{\alpha}\left(T_{\beta}(x)\right)-T_{\alpha \beta}(x),
$$

for each fixed degree $k$ of $(x-1)^{k}$, is a polynomial in $\alpha, \beta$ which vanishes, by (6.17), for all $\alpha, \beta \in \mathbb{N} \times \mathbb{N}$. Therefore, this polynomial is identically zero.

In particular, since $T_{1}(x)=x=1+(x-1)$ plays the role of the identity in the group $\left\{T_{\alpha}\right\}$, we have:

$$
\left(T_{\alpha}\right)^{-1}=T_{1 / \alpha}
$$

Most of the classical formulae for Chebyshev polynomials survive the constructed extension - either by the argument given above to prove formula (6.39a), or by observing that

$$
T_{\alpha}(x)=F\left(-\alpha, \alpha, \frac{1}{2}, \frac{1-x}{2}\right), \quad \forall \alpha .
$$

Remark 6.41. The theme of parameter extension is quite common in mathematics. The methods for constructing such extensions vary wildly. I chose in this Section the group 
property of $T_{n}$ 's. A more direct approach is to extend the parameters' meaning in the hypergeometric representations, as in formulae (6.23) and (6.40). Then there are various relations one can extend. For example, the Legendre polynomials $P_{n}(x)$ enter into the following relations with the $T_{n}(x)$ 's $([14$, p. 261]):

$$
\begin{aligned}
& \left(n+\frac{1}{2}\right)(1+x)^{1 / 2} \int_{-1}^{x} P_{n}(t)(x-t)^{-1 / 2} d t=T_{n}(x)+T_{n-1}(x), \\
& \left(n+\frac{1}{2}\right)(1-x)^{1 / 2} \int_{x}^{1} P_{n}(t)(t-x)^{-1 / 2} d t=T_{n}(x)-T_{n+1}(x),
\end{aligned}
$$

where $|x|<1, n \in \mathbb{N}$, and the integrals are taken as Cauchy principal values.

Remark 6.43. Treatises and collections of formulae on special functions should always be read with a pen, pencil, and salt shaker ready. For example, the book [9] lists on p. 206 the following formula:

$$
U_{n+1}(x)=(1-x)^{1 / 2} \sum_{k=0}^{\lfloor n / 2\rfloor} \frac{(-1)^{k}(n-k) !}{k !(n-2 k) !}(2 x)^{n-2 k} .
$$

Misprints are widespread, common, and not unexpected. Some errors are more subtle, and propagate from one book to another for many years. (See an example of 186 years old errors in [20]).

Remark 6.45. The Chebyshev polynomials $\left\{T_{n}(x)\right\}$ and the system $\left\{x^{n}\right\}$ are essentially the only two polynomial systems satisfying the semigroup/group law (see $[18,4]$ ). More general systems, if any exist, could be found only in series. For example, if one restricts oneself to the hypergeometric (classical or basic) groups, one looks for triples of functions

$$
\begin{aligned}
& a(x)={ }_{p} \mathcal{F}_{q}\left(\ldots ; \omega_{1}(x-1)\right), \\
& b(x)={ }_{r} \mathcal{F}_{s}\left(\ldots ; \omega_{2}(x-1)\right), \\
& c(x)={ }_{P} \mathcal{F}_{Q}(\ldots ; \Omega(x-1)),
\end{aligned}
$$

such that

$$
a(b(x))=c(x) .
$$

Remark 6.47. Suppose one quantizes the Chebyshev polynomials $U_{n}(x)$ by any of the standard methods of $q$-hypergeometric (= basic) series (see $[2,5,6,8]$.) Will then the sequences $S_{n}$ and $S_{n}(q)$ constructed in $\S 2$ appear as "values at $x=1 / 2$ " of the quantized polynomials? I don't see how, and think it is very unlikely, as one glance at formulae (5.25) and (5.27) will show. There is a deep mystery hidden here.

Remark 6.48. The proper meaning of the Chebyshev polynomials, as the generators of a semigroup of polynomical maps of a vector space, has been discovered by Veselov [22, 23] and by Hoffman and Withers [26, 10], with such semigroups parametrized by affine Weyl groups. It would be very interesting to find the analogs of the one-dimensional point $x=1 / 2$ and periodic sequences, both classical and quantum, for this more general set-up. 
I hope that posterity will judge me kindly, not only as to the things which I have explained, but also to those which I have intentionally omitted so as to leave to others the pleasure of discovery.

Descartes

\section{Acknowledgements}

I thank Alexander Veselov for very interesting comments and the anonymous referee for useful suggestions.

\section{References}

[1] Andrews G E, Euler's Pentagonal Number Theorem, Math. Mag. 56 (1983), 279-284.

[2] Andrews G E, Askey R and Roy R, Special Functions, Cambridge University Press, New York, 1999.

[3] Bateman H and Erdélyi A, Higher Transcendental Functions, Vol. II, McGraw-Hill Book Company, New York, 1953.

[4] Block H D and Thielman H P, Commutative Polynomials, Quart. J. Math., Oxford Ser. (2) 2 (1951), 241-243.

[5] Exton H, $q$-Hypergeometric Functions and Applications, John Wiley \& Sons, New York, 1983.

[6] Fine N J, Basic Hypergeometric Series and Applications, Amer. Math. Soc. Providence, 1988.

[7] Franco B J O and Zumpano A, Divisibility of the Coefficients of Chebyshev Polynomials by Primes, Fib. Quart. 39 (2001), 304-308.

[8] Gasper G and Rahman M, Basic Hypergeometric Series, Cambridge Univ. Press, Cambridge, 1990.

[9] Guo D R and Wang Z X, Special Functions, World Scientific, Singapore, 1989.

[10] Hoffman M E and Withers W D, Generalized Chebyshev Polynomials Associated with Affine Weyl Groups, Trans. Amer. Math. Soc. 308 (1988), 91-104.

[11] Jeffrey A, Handbook of Mathematical Formulas and Integrals, Academic Press, New York, 1995.

[12] Kupershmidt B A, q-Newton Binomial: From Euler to Gauss, J. Nonlin. Math. Phys. 7 (2000), 1-10.

[13] Kupershmidt B A, ... And Free Lunch for All. A Review of Bruce C Berndt's Ramanujan's Notebooks, Parts I-V, J. Nonlin. Math. Phys. 7 (2001), R7-R37.

[14] Magnus W, Oberhettinger F and Soni R P, Formulas and Theorems for the Special Functions of Mathematical Physics, Springer-Verlag, New York, 1966.

[15] Polya G, Induction and Analogy in Mathematics, Princeton Univ. Press, Princeton, 1954. 
[16] Pólya G and Szegö G, Problems and Theorems in Analysis, Vol. 1, Springer-Verlag, New York, 1972.

[17] Rankin R A, Chebyshev Polynomials and the Modulary Group of Level p, Math. Scand. 2 (1954), 315-326.

[18] Ritt J F, Permutable Rational Functions, Trans. Amer. Math. Soc. 25 (1923), 399-448.

[19] Rivlin T J, An Introduction to the Approximation of Functions, Dover Publications, New York, 1981.

[20] Talvila E, Some Divergent Trigonometric Integrals, Amer. Math. Monthly 108 (2001), 432436.

[21] Vasil'ev N and Zelevinskii A, Chebyshev Polynomials and Recurrence Relations, in Kvanta Selecta: Algebra and Analysis II, Editor: Tabachnikov S, Amer. Math. Soc., Providence, 1999, 51-61,

[22] Veselov A P, Integrable Mappings and Lie Algebras, Dokl. Akad. Nauk SSSR 292 (1987), 1289-1291 (in Russian); Sov. Math. Dokl. 35 (1987), 211-213.

[23] Veselov A P, Integrable Mappings, Uspekhi Mat. Nauk 46, Nr. 5 (1991), 3-45, 190 (in Russian); Russian Math. Surveys 46 (1991), 1-51.

[24] Weil A, Number Theory: an Approach Through History, from Hammurapi to Legendre, Birkhäuser, Boston, 1983.

[25] Wilf H S, Generatingfunctionology, Second ed., Academic Press, New York, 1994.

[26] Withers W D, Folding Polynomials and Their Dynamics, Amer. Math. Monthly 95 (1988), 399-413.

[27] Yantarov I, Commuting Polynomials, in Kvanta Selecta: Algebra and Analysis II, Editor: Tabachnikov S, Amer. Math. Soc., Providence, 1999, 31-38. 